\title{
Diversidad de peces en los arrecifes de coral de la costa sur-central de Cuba
}

\author{
Diversity of coral reef fish along \\ the south-central coast of Cuba
}

\author{
Alexis E. Medina Valmaseda ${ }^{1}$, José Blas Pérez Silva ${ }^{1}$, Héctor M. Salvat-Torres ${ }^{2 *}$ y Miguel \\ Salvat Quesada
}

\begin{abstract}
RESUMEN
En este trabajo se presenta el primer inventario de la ictiofauna de la costa sur-central de Cuba. El estudio fue llevado a cabo en el período entre julio de 2002 y diciembre de 2011, con el objetivo de elaborar el primer listado de la ictiofauna marina del área. La identificación de los peces se efectuó principalmente in situ, por medio de buceo autónomo, siguiendo el método de buzo errante hasta una profundidad máxima de $40 \mathrm{~m}$. La realización de buceos nocturnos facilitó la observación de las especies noctámbulas. Durante el período de estudio se realizaron un total de 195 censos diurnos y 59 censos nocturnos, 198 capturas. La lista presentada en este trabajo contiene 201 especies de peces de la clase Actinopterygii, lo que representa el 19\% de los peces pertenecientes a dicha clase registrados para Cuba. Las familias más diversas fueron: Serranidae, Apogonidae y Labridae. Se localizaron dos lugares de crianza para dos especies de pargos y una de meros. Frecuentemente se observaron individuos de la especie invasora Pterois volitans. Se presentan tres nuevos registros de especies para la región sur-central de Cuba. La diversidad de peces óseos del área estudiada es una de las mayores observadas para la región sur de Cuba, solo superada por la ictiofauna de la reserva marina de Jardines de la Reina.
\end{abstract}

Palabras claves: Peces Actinopterygii, lista de especies, nuevos registros, costa sur, Cuba.

\begin{abstract}
This paper presents the first ichthyofauna inventory for the south-central coast of Cuba. The study was conducted from July 2002 to December 2011, with the objective of making the first list of marine ichthyofauna in the area. Fish identification was carried out mainly in situ, by means of SCUBA diving following the Random Swim Technique up to a maximum depth of $40 \mathrm{~m}$. Night dives facilitated the observation of species with nocturnal habits. During the study period a total of 195 diurnal and 59 nocturnal census survey and 198 capture were conducted. The list presented in this paper has 201 fish species of the Class Actinopterygii, which represents $19 \%$ of the fish belonging to this Class in Cuba. The most diverse families were Serranidae, Apogonidae, and Labridae. Two nursery areas were located for two species of snappers and one species of groupers. Individuals of the invasive species Pterois volitans were frequently observed. Three new species are presented for the south-central region of Cuba. The diversity of bony fish in the study area is one of the richest in the southern region of Cuba, only exceeded by the diversity of ichthyofauna in the marine protected reserve Jardines de la Reina.
\end{abstract}

Keywords: Actinopterygii, fish checklist, new records, South coast, Cuba.

\footnotetext{
1 Centro de Servicios Ambientales, CITMA Sancti Spíritus, Calle Bartolomé Masó S/N, esquina Ave. de los Mártires, Sancti Spíritus, Cuba.

2 Centro de Investigaciones de Ecosistemas Costeros, Cayo Coco, Ciego de Ávila, Cuba. Email: hector@ciec.fica.inf.cu* 3 Universidad José Martí, Sancti Spíritus.
}

Recibido: 14 de mayo de 2013

Corregido: 27 de mayo de 2014

Aceptado: 11 de julio de 2014

DOI: http://dx.doi.org/10.15359/revmar.6.6 


\section{INTRODUCCIÓN}

Los estudios ecológicos de comunidades y ecosistemas requieren del conocimiento de la diversidad de especies claves (Pina-Amargós et al. 2007). Los peces se encuentran en este grupo por su papel modulador a través de la depredación, el herbivorismo, la bioerosión y otros aspectos vinculados a la actividad trófica. Además, la importancia de los peces es reforzada por ser el objeto primordial de la actividad pesquera, una de las principales actividades humanas en los océanos (Freon \& Misund, 1999).

La zona costera del macizo de Guamuhaya se encuentra en la región sur-central de Cuba y se caracteriza por ser una costa sin plataforma, que separa físicamente dos importantes golfos: Ana María y Batabanó (Montero, 2000). Este litoral presenta terrazas marinas de fondo duro, con arrecifes de coral bien conservados y altamente apreciados por la actividad de buceo recreativo. Sin embargo, su biota marina ha sido poco estudiada. El área de estudio colinda con dos zonas costeras de importantes valores ecológicos, pesqueros y turísticos. Los arrecifes de coral del Parque Nacional Jardines de la Reina, ubicado en el golfo de Ana María, son considerados entre los de mayor diversidad en el Caribe (Pina-Amargós et al. 2007), mientras que el golfo de Batabanó presenta áreas importantes de agregación para el desove de pargos y meros, como es el Banco de Jagua (Claro \& Lindeman, 2003), así como las mayores capturas de langosta espinosa (Panulirus argus).
La lista más reciente de la ictiofauna cubana consigna la presencia de 1120 especies (Claro \& Robertson, 2010). De las especies estrictamente marinas 85 pertenecen a la clase Chondrichthyes y 1030 a la clase Actinopterygii; los 5 restantes son peces óseos dulceacuícolas. Las familias de peces óseos con mayor diversidad de especies en aguas cubanas son: Serranidae, Carangidae, Lutjanidae, Labridae, Labrisomidae, Gobiidae y Haemulidae. La mayor riqueza de especies se presenta en el orden Perciformes, en el cual se registran hasta ahora 60 familias, 245 géneros y 486 especies. A juzgar por los límites de distribución de las especies de peces reportadas en el Gran Caribe, y que no están registradas para Cuba, se estima que al menos unas 85 especies más podrían estar presentes en aguas cubanas (Claro, 2007).

En este artículo se presenta por primera vez la lista de especies de peces óseos de los arrecifes de coral de la zona sur-central de Cuba. Los resultados de este trabajo son la base para futuros estudios científicos, así como para el manejo de los recursos pesqueros y la conservación de la biodiversidad en la zona.

\section{MATERIALES Y MÉTODOS}

La zona costera del macizo de Guamuhaya está ubicada en la región sur-central de Cuba, compartida por las provincias de Sancti Spíritus y Cienfuegos. Está conformada por una zona cárstica, donde se intercalan playas arenosas de longitudes variables 
con escurrimiento de agua dulce provenientes de las montañas. La parte sumergida se caracteriza por poseer una plataforma estrecha de entre $200 \mathrm{y}$ $800 \mathrm{~m}$, con una franja de arrecifes de coralinos en el borde de la terraza marina. Entre esta zona y la orilla está presente un área de terraza rocosa abrasiva o zona de Pseudopterogorgia (ramajal) de fondos duros, colonizados por arrecifes de parche e interrumpida por pequeños parches del erizo de mar Echinometra. La profundidad varía entre 1.5 y $6 \mathrm{~m}$, hasta llegar a los 15 y $20 \mathrm{~m}$ en la parte superior del escarpe arrecifal. Después de los $20 \mathrm{~m}$ de profundidad, el escarpe presenta una caída vertical en forma de pared que sobrepasa los $200 \mathrm{~m}$. La pared del escarpe está compuesta por grandes grietas cubiertas de corales pétreos. El área de trabajo tuvo una extensión lineal aproximada de $8 \mathrm{~km}$ y se enmarcó entre la zona de la desembocadura del río Cabagán $\left(21^{\circ} 49^{\prime} \mathrm{N}\right.$ y $80^{\circ}$ 06’ W) y un sector de la península de Ancón conocido como Las Caletas ( $21^{\circ} 46^{\prime} \mathrm{N}$ y $80^{\circ} 01^{\prime} \mathrm{W}$ ) (Fig. 1).

Los censos se realizaron en el período comprendido entre julio de 2002 y diciembre de 2011 por un equipo de cuatro buzos, dos especialistas en peces y dos aseguradores. La frecuencia de trabajo fue bimensual por cada año de muestreos. Los sitios de trabajo no fueron siempre los mismos en cada año, para lograr una mayor área de trabajo censada (Fig. 1). Cada censo fue realizado paralelo a la costa,

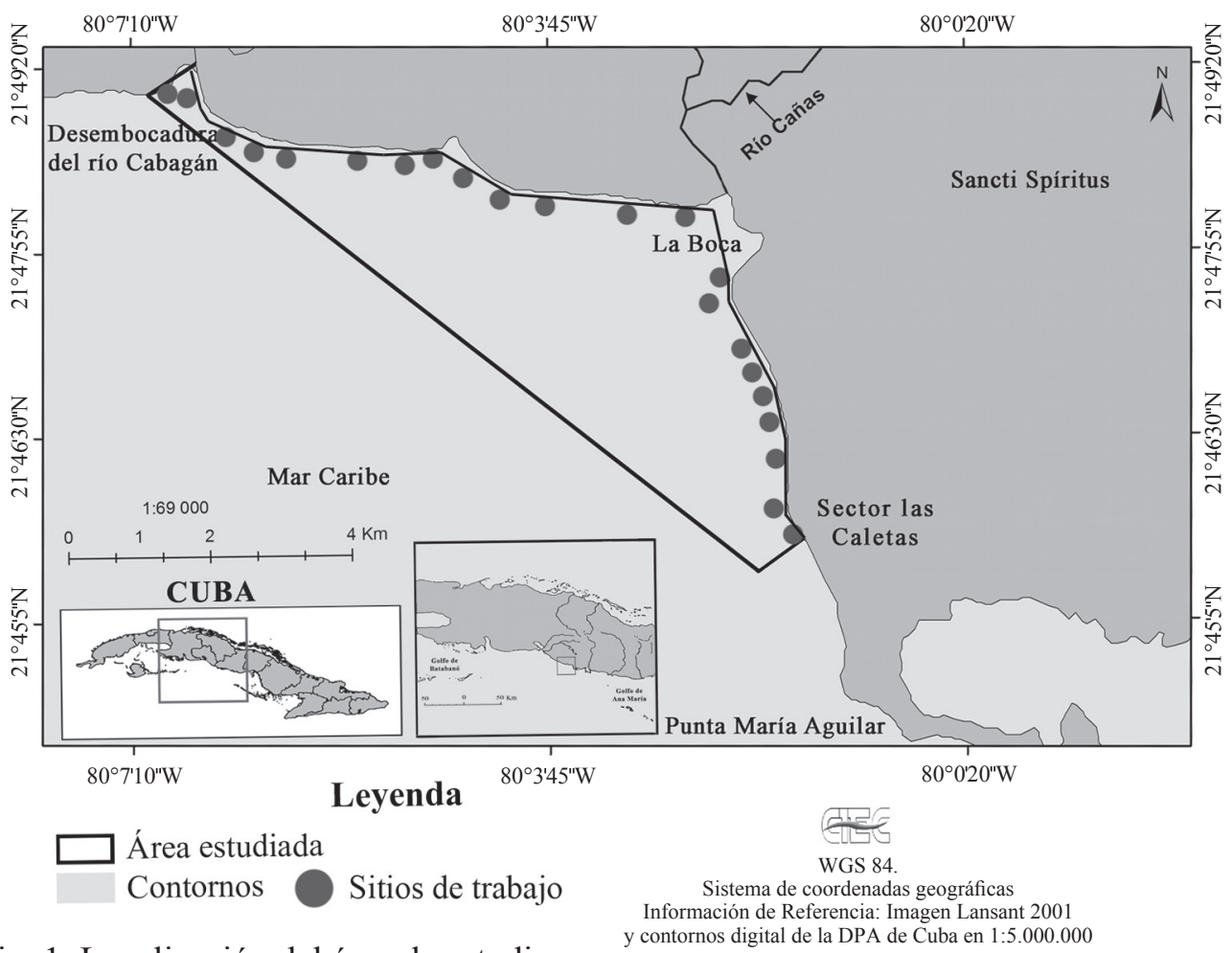

Fig. 1. Localización del área de estudio y contornos digital de la DPA de Cuba en 1:5.000.000

Fig. 1. Location of the study area 
siguiendo el método de censo de buzo errante, durante $50 \mathrm{~min}$, mediante buceo autónomo (SCUBA) (Jones \& Thompson, 1978). Este método permite obtener estimaciones de la riqueza de especie con mayor exactitud que el resto de los métodos usados para peces (Bortone et al. 1986).

Para el conteo de las especies se abarcaron todos los hábitats posibles, desde los profundos como el escarpe arrecifal (15$20 \mathrm{~m}$ ) y la pared del escarpe ( $25-40 \mathrm{~m})$, hasta los sectores someros como la parte frontal del escarpe arrecifal (3-5 m). Por cada sitio de trabajo se realizaron buceos por las noches, lo que facilitó la observación y captura de especies noctámbulas; dos censos visuales, anotando todas las especies de peces observadas y examinando cuidadosamente grietas, esponjas, parches de arena o cualquier refugio posible para los peces.

La identificación de las especies se realizó principalmente in situ, las especies de identificación dudosa solo fueron incluidas en la lista cuando fueron capturadas y observadas por dos o más autores y siguiendo las claves de identificación. No se incluyeron las especies obtenidas de las pesquerías ni ninguna otra cuya presencia en la zona no fuera verificada por los autores. En el caso de Pterois volitans su identificación se realizó en laboratorio, para confirmar que fuera esa especie y no $P$. miles. Para la determinación de los peces se utilizaron las obras de Guitart (1985), Robins \& Ray (1986), Bölke \& Chaplin (1993), Randall (1996) y Humann \& Deloach (2006). Para la organización supragenérica de las especies se utilizó el criterio de Nelson (2006) y para los géneros y especies, el de Eschmeyer (2013)

\section{RESULTADOS}

Durante los 254 censos y 198 capturas realizadas en los arrecifes de coral de la costa del macizo de Guamuhaya se identificaron 201 especies pertenecientes a 43 familias y 8 órdenes de la clase Actinopterygii. Las familias más diversas fueron: Serranidae, Labridae, Pomacentridae, Apogonidae, Scaridae, Lutjanidae y Carangidae. Las especies que se observaron con mayor frecuencia fueron: Stegastes partitus (Poey, 1868), Chromis cyanea (Poey, 1860), Thalassoma bifasciatum (Bloch, 1791), Halichoeres garnoti (Valenciennes, 1839), Serranus tabacarius (Cuvier, 1829), Acanthurus coeruleus Bloch y Schneider, 1801, Acanthurus tractus Poey, 1860, Haemulon sciurus (Shaw, 1803), Sparisoma aurofrenatum (Valenciennes, 1840) y el complejo $S$. iseri/ taeniopterus. Estos últimos fueron observados principalmente en su fase juvenil en casi todas las zonas de buceo, aunque no en todos los biotopos.

En el 95\% de los sitios de trabajo fue observada la especie invasora Pterois volitans (Linnaeus, 1758). También es de destacar la presencia de grupos numerosos de Lonchopisthus micrognathus (Poey, 1860) en profundidades de 15 a $19 \mathrm{~m}$. Los censos y capturas realizados en horas de la noche permitieron observar las especies con hábitos nocturnos, como son los miembros de la familia Apogonidae (cardenales). Estas especies son difíciles de detectar durante el día, ya que permanecen 
ocultas en grietas y oquedades. En el cuadro 1 se muestra la lista sistemática de los taxones identificados.

Cuadro 1. Lista de especies de peces identificados en la región sur-central de Cuba. $*=$ nuevo registro para la zona

Table 1. List of fish species identified in southcentral Cuba. ${ }^{*}=$ new record for the area

Clase Actinopterygii

Orden Anguilliformes

Familia Congridae Kaup 1856

Heteroconger longissimus Günther, 1870

Familia Ophichthidae Günther 1870

Echiophis intertinctus (Richardson, 1848)

Myrichthys breviceps (Richardson, 1848)

Familia Muraenidae Rafinesque 1810

Echidna catenata (Bloch, 1795)

Gymnothorax funebris Ranzani, 1839

Gymnothorax miliaris (Kaup, 1856)

Gymnothorax moringa (Cuvier, 1829)

Gymnothorax vicinus (Castelnau, 1855)

Orden Aulopiformes

Familia Synodontidae Gill 1861

Synodus intermedius (Spix y Agassiz, 1829)

Orden Perciformes

Familia Serranidae Swainson 1839

Cephalopholis cruentata (Lacepède, 1802)

Cephalopholis fulva (Linnaeus, 1758)

Epinephelus adscensionis (Osbeck, 1765)

Epinephelus guttatus (Linnaeus, 1758)

Epinephelus itajara (Lichtenstein, 1822)

Epinephelus morio (Valenciennes, 1828)

Hyporthodus mystacinus (Poey, 1852)

Epinephelus striatus (Bloch, 1792)

Hypoplectrus aberrans Poey, 1868

Hypoplectrus chlorurus (Cuvier, 1828)

Hypoplectrus gummigutta (Poey, 1851)

\section{DISCUSIÓN}

Este es el primer estudio que se realiza en esta zona sobre los

Hypoplectrus guttavarius (Poey, 1852)

Hypoplectrus indigo (Poey, 1851)

Hypoplectrus nigricans (Poey, 1852)

Hypoplectrus puella (Cuvier, 1828)

Hypoplectrus unicolor (Walbaum, 1792)

Liopropoma mowbrayi Woods y Kanazawa, 1951

Liopropoma rubre Poey, 1861

Mycteroperca bonaci (Poey, 1860)

Mycteroperca interstitialis (Poey, 1860)

Mycteroperca tigris (Valenciennes, 1833)

Mycteroperca venenosa (Linnaeus, 1758)

Rypticus saponaceus (Bloch y Schneider, 1801)

Rypticus subbifrenatus Gill, 1861

Serranus baldwini (Evermann y Marsh, 1899)

Serranus subligarius (Cope, 1870) *

Serranus tabacarius (Cuvier, 1829)

Serranus tigrinus (Bloch, 1790)

Serranus tortugarum Longley, 1935

Familia Lutjanidae Gill 1862

Lutjanus analis (Cuvier, 1828)

Lutjanus apodus (Walbaum, 1792)

Lutjanus buccanella (Cuvier, 1828)

Lutjanus jocu (Bloch y Schneider, 1801)

Lutjanus mahogoni (Cuvier, 1828)

Lutjanus purpureus (Cuvier, 1828)

Lutjanus synagris (Linneaus, 1758)

Ocyurus chrysurus (Bloch, 1791)

Familia Haemulidae Gill 1885

Anisotremus virginicus (Linnaeus, 1758)

Haemulon album Cuvier, 1830

Haemulon aurolineatum Cuvier, 1830

Haemulon carbonarium Poey, 1860

Haemulon chrysargyreum Günther, 1859

Haemulon flavolineatum (Desmarest, 1823) 
Haemulon plumieri (Lacepède, 1801)

Haemulon sciurus (Shaw, 1803)

Haemulon striatum (Linnaeus, 1758)

Familia Sparidae Rafinesque 1810

Calamus bajonado (Bloch y Schneider, 1801)

Calamus calamus (Valenciennes, 1830)

Familia Sciaenidae Cuvier 1828

Equetus acuminatus (Bloch y Schneider, 1801)

Equetus lanceolatus (Linnaeus, 1758)

Equetus punctatus (Bloch y Schneider, 1801)

Familia Pempheridae Gill 1862

Pempheris schomburgkii (Müller y Troschel, 1848)

Familia Chaetodontidae Rafinesque 1810

Prognathodes aculeatus (Poey, 1860)

Chaetodon capistratus Linnaeus, 1758

Chaetodon ocellatus Bloch, 1787

Chaetodon sedentarius Poey, 1860

Chaetodon striatus Linnaeus, 1758

Familia Pomacanthidae Cockerell 1913

Centropyge argi Woods y Kanazawa, 1951

Holacanthus ciliaris (Linnaeus, 1758)

Holacanthus tricolor (Bloch, 1795)

Pomacanthus arcuatus (Linnaeus, 1758)

Pomacanthus paru (Bloch, 1787)

Familia Kyphosidae Gill 1893

Kyphosus incisor (Cuvier, 1831)

Kyphosus sectatrix (Linnaeus, 1758)

Familia Cirrhitidae Macleay 1841

Amblycirrhitus pinos (Mowbray, 1927)

Familia Pomacentridae Bonaparte 1831

Abudefduf saxatilis (Linnaeus, 1758)

Abudefduf taurus (Müller y Troschel, 1848)

Chromis cyanea (Poey, 1860)

Chromis enchrysura Jordan y Gilbert, 1882

Chromis insolata (Cuvier, 1830)

Chromis multilineata (Guichenot, 1853)

Chromis scotti Emery, 1968 *
Microspathodon chrysurus (Cuvier, 1830)

Stegastes diencaeus (Jordan y Rutter, 1897)

Stegastes leucostictus (Müller y Troschel, 1848)

Stegastes partitus (Poey, 1868)

Stegastes planifrons (Cuvier, 1830)

Stegastes adustus (Troschel, 1865)

Familia Sphyraenidae Rafinesque 1815

Sphyraena barracuda (Walbaum, 1792)

Sphyraena borealis De Kay, 1842

Sphyraena guachancho Cuvier, 1829

Familia Scombridae Rafinesque 1810

Sarda sarda (Bloch, 1793)

Scomberomorus regalis (Bloch, 1793)

Familia Grammatidae Jordan y Evermann 1896

Gramma linki Starck y Colin, 1978

Gramma loreto Poey, 1868

Gramma dejongi Victor y Randall, 2010)

Gramma melacara Böhlke y Randall, 1963

Familia Opistognathidae Bonaparte 1835

Lonchopisthus micrognathus (Poey,1860)*

Opistognathus aurifrons (Jordan y Thompson, 1905)

Opistognathus macrognathus (Poey, 1860)

Opistognathus lonchurus Jordan y Gilbert, 1882

Opistognathus maxillosus Poey, 1860

Familia Priacanthidae Günther 1859

Heteropriacanthus cruentatus (Lacepède, 1801)

Familia Apogonidae Günther 1859

Apogon affinis (Poey, 1875)

Apogon aurolineatus (Mowbray, 1927)

Apogon binotatus (Poey, 1867)

Apogon lachneri Böhlke, 1959

Apogon maculatus (Poey, 1860)

Apogon phenax Böhlke y Randall, 1968

Apogon planifrons Longley y Hildebrand, 1940

Apogon pseudomaculatus Longley, 1932 
Apogon quadrisquamatus Longley, 1934 Apogon robinsi Böhlke y Randall, 1968 Apogon townsendi (Breder, 1927) Astrapogon puncticulatus (Poey, 1867) Phaeoptyx pigmentaria (Poey, 1860)

Familia Malacanthidae Günther 1861 Malacanthus plumieri (Bloch, 1786)

Familia Echeneidae Rafinesque 1810 Echeneis naucrates Linnaeus, 1758 Remora remora (Linnaeus, 1758)

Familia Rachycentridae Jordan y Evermann 1896

Rachycentron canadum (Linnaeus, 1766)

Familia Coryphaenidae Rafinesque 1810 Coryphaena equiselis Linnaeus, 1758

Familia Carangidae Rafinesque 1815 Alectis ciliaris (Bloch, 1787)

Caranx bartholomaei Cuvier, 1833

Caranx crysos (Mitchill, 1815)

Caranx hippos (Linnaeus, 1766)

Caranx latus Agassiz, 1831

Caranx lugubris Poey, 1860

Caranx ruber (Bloch, 1793)

Elagatis bipinnulata (Quoy y Gaimard, 1825)

Selene vomer (Linnaeus, 1758)

Familia Labridae Cuvier 1816

Bodianus rufus (Linneaus, 1758)

Clepticus parrae (Bloch y Schneider, 1801)

Halichoeres bivittatus (Bloch, 1791)

Halichoeres garnoti (Valenciennes, 1839)

Halichoeres maculipinna (Müller y Troschel, 1848)

Halichoeres pictus (Poey, 1860)

Halichoeres poeyi (Steindachner, 1867)

Halichoeres radiatus (Linnaeus, 1758)

Lachnolaimus maximus (Walbaum, 1792)

Thalassoma bifasciatum (Bloch, 1791)

Xyrichtys martinicensis Valenciennes, 1840
Xyrichtys novacula (Linnaeus, 1758)

Xyrichtys splendens Castelnau, 1855

Familia Scaridae Rafinesque 1810

Scarus guacamaia Cuvier, 1829

Scarus iseri (Bloch, 1789)

Scarus taeniopterus Lesson 1829

Scarus vetula Bloch y Schneider, 1801

Sparisoma aurofrenatum (Valenciennes, 1840)

Sparisoma chrysopterum (Bloch y Schneider, 1801)

Sparisoma radians (Valenciennes, 1840)

Sparisoma rubripinne (Valenciennes, 1840)

Sparisoma viride (Bonnaterre, 1788)

Familia Labrisomidae Clark Hubbs 1952 Labrisomus filamentosus Springer, 1960 * Labrisomus nigricinctus Howell Rivero, 1936 Labrisomus nuchipinnis (Quoy y Gaimard, 1824) Malacoctenus aurolineatus Smith, 1957 Malacoctenus boehlkei Springer, 1959 Malacoctenus triangulatus Springer, 1959

Familia Chaenopsidae Gill 1865 Acanthemblemaria aspera (Longley, 1927) Acanthemblemaria maria Böhlke, 1961 Acanthemblemaria spinosa Metzelaar, 1919 Emblemaria pandionis Evermann y Marsh, 1900 Lucayablennius zingaro (Böhlke, 1957)

Familia Blenniidae Rafinesque 1810 Parablennius marmoreus (Poey, 1876) Scartella cristata (Linnaeus, 1758) Ophioblennius macclurei (Silvester, 1915)

Familia Gobiesocidae Bleeker 1859 Acyrtus rubiginosus (Poey, 1868) Gobiesox punctulatus (Poey, 1876)

Familia Gobiidae Cuvier 1816 Elacatinus dilepis (Robins y Böhlke, 1964) Elacatinus evelynae (Böhlke y Robins, 1968) Elacatinus multifasciatus (Steindachner, 1876) Elacatinus horsti (Metzelaar, 1922) 
Lophogobius cyprinoides (Pallas, 1770)

Nes longus (Nichols, 1914)

Coryphopterus glaucofraenum Gill,1863

Coryphopterus hyalinus Böhlke y Robins, 1962

Familia Acanthuridae Bonaparte 1835

Acanthurus tractus Poey, 1860

Acanthurus chirurgus (Bloch, 1787)

Acanthurus coeruleus Bloch y Schneider, 1801

Orden Pleuronectiformes

Familia Bothidae Regan 1910

Bothus lunatus (Linnaeus, 1758)

Bothus ocellatus (Agassiz, 1831)

Orden Dactylopteriformes

Familia Dactylopteridae Gill 1861

Dactylopterus volitans (Linnaeus, 1758)

Orden Scorpaeniformes

Familia Scorpaenidae Risso 1827

Pterois volitans Linnaeus, 1758

Scorpaena plumieri Bloch, 1789

Scorpaena inermis Cuvier, 1829

Orden Syngnathiformes

Familia Syngnathidae Rafinesque 1810

Cosmocampus elucens (Poey, 1868)

Micrognathus crinitus (Jenyns, 1842)

Orden Tetraodontiformes

Familia Balistidae Rafinesque 1810

Balistes vetula Linnaeus, 1758

Melichthys niger (Bloch, 1786)

Familia Monacanthidae Nardo 1843

Aluterus scriptus (Osbeck, 1765)

Cantherhines macrocerus (Hollard, 1853)

Cantherhines pullus (Ranzani, 1842)

Monacanthus tuckeri Bean, 1906

Familia Ostraciidae Rafinesque 1810

Lactophrys bicaudalis (Linnaeus, 1758)

Rhinesomus triqueter (Linnaeus, 1758)

Canthigaster rostrata (Bloch, 1786)

Familia Tetraodontidae Bonaparte 1831
Sphoeroides spengleri (Bloch, 1785)

Sphoeroides testudineus (Linnaeus, 1758)

Familia Diodontidae Bonaparte 1835

Chilomycterus antennatus (Cuvier, 1816)

Chilomycterus antillarum Jordan y Rutter, 1897

Diodon holocanthus Linnaeus, 1758

Diodon hystrix Linnaeus, 1758

peces marinos, por lo que no se tiene conocimiento de datos anteriores. Según la lista de peces publicada en este trabajo, en la costa del macizo de Guamuhaya está representado el 19\% de los peces pertenecientes a la clase Actinopterygii registrados para Cuba, tomando como referencia a Claro \& Robertson (2010). Teniendo en cuenta que para este trabajo solo fueron censados los peces óseos, el número de especies es elevado, solo superado por la lista de especies del Parque Nacional Jardines de la Reina con 264 peces de la clase Actinopterygii, publicada por Pina-Amargós et al. (2012). Llama la atención que la diversidad de peces en la costa del macizo de Guamuhaya es también mayor que la de las regiones norte-occidental (González-Sansón et al. 1997; Aguilar et al. 2000) y norte centro-oriental (Lara-Lorenzo et al. 2013) de Cuba (Fig. 2). Estas diferencias pueden deberse a las características propias de cada una de las regiones estudiadas, así como a su grado de protección y no al esfuerzo de muestreo, ya que este fue similar en cada estudio $(\mathrm{n}=200$ Pina-Amargós et al. 2012; $\mathrm{n}=201$ González-Sansón et al. 1997; $\mathrm{n}=198$ Aguilar et al. 2000; $\mathrm{n}=225$ Lara-Lorenzo et al. 2013 y n = 209 en este trabajo). 


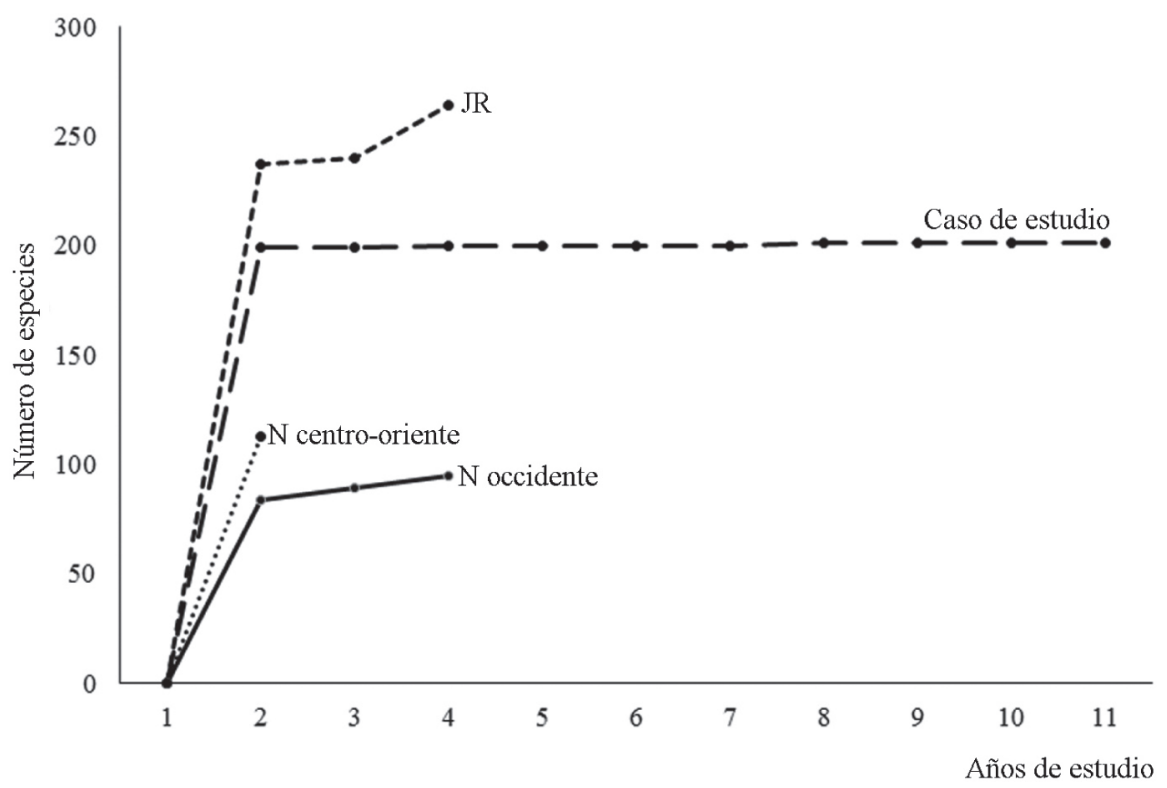

Fig. 2. Curvas acumulativas del número de especies por años de estudio en cuatro zonas marinas de Cuba. $\mathrm{JR}=$ Jardines de la Reina

Fig. 2. Cumulative curves of the number of species by years of study in four marine areas in Cuba. JR= Jardines de la Reina

La alta diversidad de peces de arrecifes encontrados en la costa del macizo de Guamuhaya está asociada a dos situaciones. Según la literatura, la mayor diversidad de peces se observa en los arrecifes de coral, principalmente en las pendientes y crestas arrecifales (Claro et al. 2004). Mientras que la segunda situación está dada por la condición estrecha de la plataforma marina en la zona estudiada. Ello propicia que hábitats (cabezos, escarpe arrecifal y la pared del escarpe) con diferentes grados de complejidad estén muy cercanos entre sí, lo que mejora la conectividad de los peces entre estos. Estudios de Chittaro (2004) y Halpern (2004) han sugerido que a mayor distancia entre los hábitats que componen un sistema costero es menor el número de peces que se pueden registrar, ya que menos juveniles pueden llegar a ser adultos, como consecuencia de la depredación. La mortalidad puede ser mayor con el incremento de la distancia entre hábitats diferentes, o pueden tener problemas al encontrar un hábitat apropiado para adultos, si la distancia es muy grande entre estos dos hábitats.

El largo período de estudio permitió ubicar dos posibles áreas de crianza para individuos de la familia Lutjanidae (pargos) y Serranidae (meros), ya que durante el período de reproducción para estas familias (abril-agosto) era común observar elevadas concentraciones de juveniles de las especies Lutjanus analis (Cuvier, 1828), Lutjanus buccanella (Cuvier, 1828) e Hyporthodus mystacinus (Poey, 1852). Es importante mencionar que estas especies podrían estar 
sufriendo los impactos de las pesquerías con artes masivas que aún son utilizadas por parte de entidades estatales $o$ personas particulares. Un caso similar es documentado por González-Sansón et al. (1997) y Aguilar et al. (2000), quienes hacen referencia a la escasez y en algunos casos a la ausencia de los depredadores grandes (familias: Serranidae, Lutjanidae, Sphyraenidae y Carangidae y tiburones), lo que se atribuye a la intensa presión pesquera en la región noroccidental de Cuba.

Las especies Serranus subligarius (Cope, 1870), Chromis scotti (Emery, 1968) y Labrisomus filamentosus (Springer, 1960) fueron registradas hace más de diez años para Cuba en la costa norte por Tallet (2001); sin embargo, con este estudio constituyen nuevos registros para la costa de la región sur-central de Cuba. Por su parte, según la literatura (Claro \& Robertson, 2010) Lonchopisthus micrognathus se encuentra a profundidades iguales $o$ mayores a $22 \mathrm{~m}$; sin embargo, en este estudio fue observada a menos de esa profundidad, específicamente a los $15 \mathrm{~m}$. Esto permite establecer un nuevo registro en la profundidad mínima de esta especie.

La lista de especies de este trabajo es de gran importancia porque permite establecer la línea base para futuros estudios comparativos en la zona. A su vez, es el primer elemento ventajoso utilizado por los gestores y responsables de la conservación de los recursos marinos, a partir del cual se pueden diseñar investigaciones para describir los procesos ecológicos que ocurren en la zona. Un estudio más amplio sobre la estructura y ecología de la comunidad de peces presentes en la costa del macizo de Guamuhaya, así como su conectividad con áreas aledañas, permitirá elaborar estrategias de manejo y conservación que aporten beneficios a este ecosistema y a las personas que de él se sirven.

\section{AGRADECIMIENTOS}

Los autores agradecen a Yudisleyvis Ventura por la realización del mapa, a Omar Fernández por la revisión del documento. Agradecemos al Ministerio de Ciencia, Tecnología y MedioAmbiente por su apoyo en el trabajo realizado.

\section{BIBLIOGRAFÍA}

Aguilar, C., González-Sansón, G., de la Guardia, E., Suárez, A. M., Trelles, J. \& Angulo, J. (2000). Inventario de los componentes más comunes de la flora y la fauna del arrecife de coral costero de la Caleta de San Lázaro, región noroccidental de Cuba, en el período de 1996 a 1998. Rev. Invest. Mar., 21(1-3), 53-59.

Bölke, J. E. \& Chaplin, C. C. G. (1993). Fishes of the Bahamas and adjacent tropical waters. Austin, Texas, EE.UU.: University of Texas Press.

Bortone, S. A., Hastings, R. W. \& Oglegby, J. L. (1986). Quantification of reef fish assemblages: A comparison of several in situ methods. Northeast Gulf Sci., 8(1), 1-22.

Chittaro, P. M. (2004). Fish-habitat associations across multiple spatial scale. Coral Reef, 23, 235-244. DOI: $\quad$ http://dx.doi.org/10.1007/ s00338-004-0376-z

Claro, R. \& García-Arteaga, J. P. (1993). Estructura de las comunidades de peces asociados a los manglares del Grupo Insular SabanaCamagüey, Cuba. Avicennia, 0, 60-83. 
Claro, R. \& García-Arteaga, J. P. (1994). Estructura de las comunidades de peces en los arrecifes del grupo insular SabanaCamagüey, Cuba. Avicennia, 2, 83-107.

Claro, R., García-Arteaga, J. P., Gobert, B., Cantelar, R. K., Valle Gómez, S. V. \& Pina Amargós, F. (2004). Situación actual de los recursos pesqueros del Archipiélago Sabana-Camagüey, Cuba. Bol. Invest. Mar. Cost., 33, 49-67.

Claro, R. \& Lindeman, K. C. (2003). Spawning aggregation sites of Snapper and Grouper species (Lutjanidae and Serranidae) on the Insular Shelf of Cuba. Gulf and Carib. Research., 14(2), 91-106.

Claro, R. (2007). II Diversidad Ecológica. En R. Claro (Ed.), La biodiversidad marina de Cuba (pp. 15-47). La Habana, Cuba: Instituto de Oceanología, Ministerio de Ciencia, Tecnología y Medio Ambiente.

Claro, R. \& Robertson, D. R. (2010). Los peces de Cuba. La Habana, Cuba: Instituto de Oceanología, CITMA.

Eschmeyer, W. N.(Ed.). (2013). Catalog of Fishes: Genera, Species. Recuperado diciembre 12, 2013, disponible http:// research.calacademy.org/research/ ichthyology/catalog/fishcatmain.asp.

Freon, P. \& Misund, O. A. (1999). Dynamics of Pelagic Fish Distribution and Behaviour: Effects on Fisheries and Stock Assessment. Oxford, Inglaterra: Fishing New Books.

González-Sansón, G., de la Guardia, E., Aguilar, C., González, C. \& Ortiz, M. (1997). Inventario de los componentes más comunes de la fauna en un arrecife de coral costero de la región noroccidental de Cuba. Rev. Invest. Mar., 18(3), 193-197.

Guitart, D. (1985). Sinopsis de los peces marinos de Cuba. La Habana, Cuba: Editorial Científico-Técnica.
Halpern, B. S. (2004). Are mangroves a limiting resource for two coral reef fishes? Mar. Ecol. Prog. Ser., 272, 9398. DOI: http://dx.doi.org/10.3354/ meps272093

Humann, P. \& Deloach, N. (2006). Reef fish identification, Florida Caribbean Bahamas. Jacksonville, EE.UU.: New World Publications, Inc.

Jones, R. S. \& Thompson, M. J. (1978). Comparison of Florida reef fish assemblages using a rapid visual technique. Bull. Mar. Sci., 28, 159-172.

Lara-Lorenzo, A., García, A., Chevalier, P., Salvat-Torres, H. \& Claro, R. (2013). Estado de conservación de la ictiofauna arrecifal en sitios de buceo turístico de cuatro regiones del Archipiélago SabanaCamagüey, Cuba. Ser. Oceanol., 13, 76-94. Montero, J. F. (2000). Diccionario geográfico de Cuba. La Habana, Cuba: GEO.

Nelson, J. S. (2006). Fishes of the World (4ª. Ed.). New Jersey, EE.UU.: John Wiley y Sons.

Pina-Amargós, F., Claro, R., García, J. P., López, N. \& González-Sansón, G. (2007). Ictiofauna del archipiélago Jardines de la Reina, Cuba. Rev. Invest. Mar., 28(3), 217-223.

Pina-Amargós, F., Salvat-Torres, H. \& López-Fernández, N. (2012). Ictiofauna del archipiélago Jardines de la Reina, Cuba. Rev. Invest. Mar., 32(2), 54-65.

Randall, J. E. (1996). Caribbean reef fishes. Jersey City, EE.UU.: T. F. H. Publications.

Robins, C. R. \& Ray, G. C. (1986). A field guide to Atlantic coastal fishes. North America. Boston, EE.UU: Houghton Mifflin.

Tallet, C. (2001). Nuevos registros para la ictiofauna cubana, con notas sobre su ecología y conducta en acuario. Rev. Invest. Mar., 22(2), 159-161. 